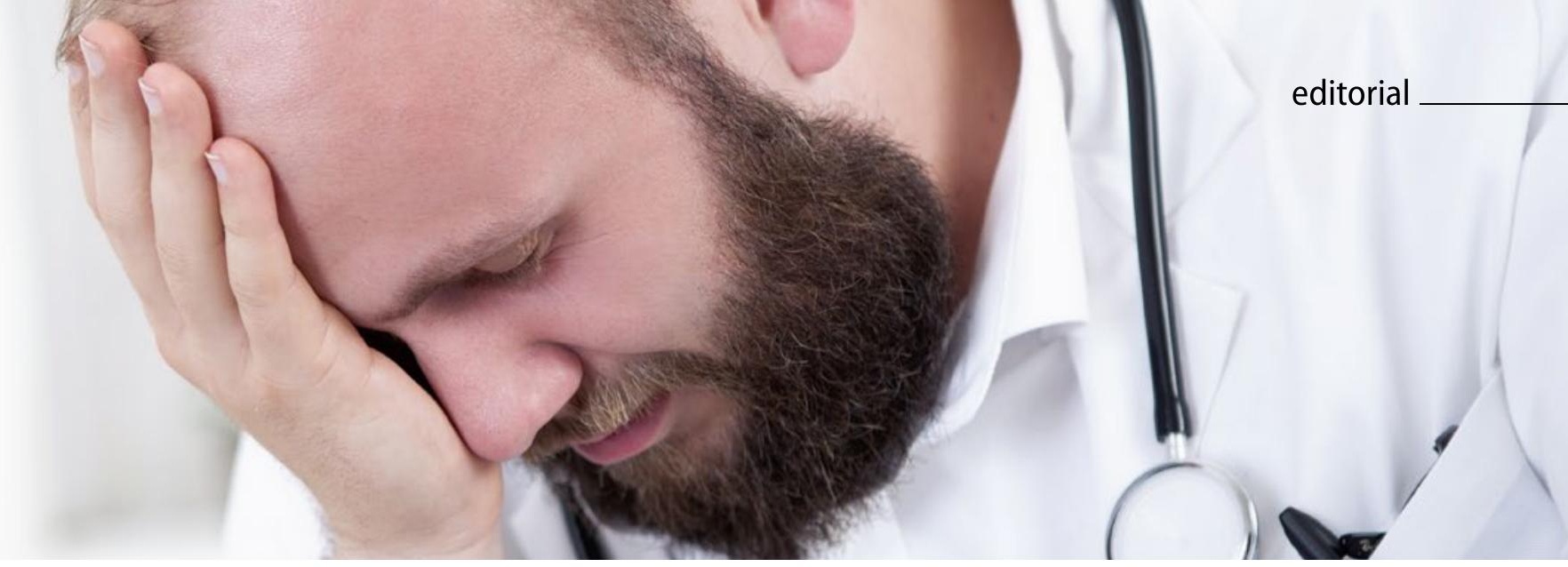

\title{
Auch Kommunikationstraining beugt vor
}

Der Begriff „Burnout“ wird nahezu inflationär benutzt. Im Wesentlichen aber geht es darum, ein Burnout zu verhindern, um die professionelle Handlungs- und Beziehungsfähigkeit, berufliche Zufriedenheit sowie körperliches wie psychisches Wohlbefinden zu fördern und zu erhalten.

Dies setzt Klarheit über den Begriff Burnout bei Ärzten, auch Onkologen, voraus. Unter beruflichem Burnout sind

- der allmähliche Verlust von Engagement, Interesse und Freude an der Arbeit (emotionale Erschöpfung),

- die innere Distanzierung, Vermeidung oder eine zynische Haltung („Depersonalisation“) zum eigenen Selbstschutz, und

- der Verlust der Überzeugung, dass die Arbeit sinnvoll und bedeutsam ist (geringe persönliche Erfüllung) zu verstehen.

Diese drei Dimensionen erfasst der „Maslach Burnout Inventory“ (MBI), der international als Goldstandard für die Selbsteinschätzung beruflicher Beanspruchung gilt; der MBI erlaubt jedoch keine klinische Diagnose von Burnout. Zeichen von Burnout wirken sich nachteilig auf die Versorgungsqualität von Patienten, auf die ärztliche Empathiefähigkeit und auf die Qualität der Arzt-Patient-Beziehung aus. Seitens der Ärzte gehen Zeichen von Burnout mit ungünstiger „Work-Life-Balance“, geringer subjektiver Lebensqualität sowie deutlich vermehrtem Risiko für Depression, Alkohol- und Substanzabusus einher.

Entgegen bisheriger Annahmen scheint aber berufliches Burnout bei onkologisch tätigen Ärzten nicht häufiger oder stärker ausgeprägt zu sein als z. B. bei Allgemeinärzten, Pädiatern oder Chirurgen. Vermutlich überwiegen fachübergreifend die Gemeinsamkeiten hinsichtlich struktureller und organisatorischer Ursachen, wie sie unter dem Primat ökonomischer Effizienz und Verknappung personeller Ressourcen (nicht nur) das deutsche Gesundheitssystem kennzeichnen: Weiter zunehmender Zeitund Effizienzdruck, während der Zeitaufwand für patientenferne Aufgaben immer weniger Zeit für Patientenkontakte lässt, gehen alle zu Lasten der Versorgungsqualität für Patienten, und garantieren steigende Burnout-Raten bei Ärzten. Das Burnout-Risiko von Onkologen war bei einer Konsultationsdauer von 20 Minuten um 36\% höher als bei 30 Minuten je Patient [Shanafelt TD et al. J Clin Oncol. 2014;32(7):678-86].

Burnout bei Onkologen ist alles andere als ein individuelles psychisches Problem; dem folglich nicht vorrangig mit individuellen psychohygienischen Strategien zur Steigerung von „Resilienz", „Hardiness“ und Produktivität beizukommen ist. Organi-

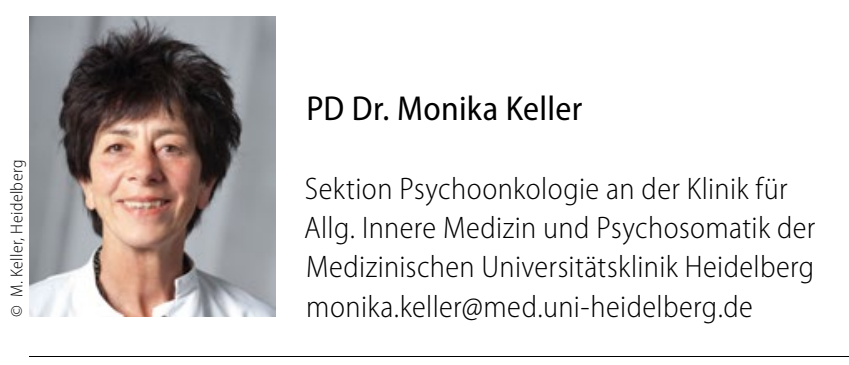

satorische und strukturelle Veränderungen, mit denen die Versorgungsqualität von Tumorpatienten ebenso wie die Arbeitsbedingungen von Onkologen verbessert werden könnten, würden jedoch einen dezidierten politischen Willen erfordern.

Viele Onkologen erleben die Arbeit mit Tumorpatienten ebenso bereichernd wie beanspruchend. In der alltäglichen Konfrontation mit Leiden und Tod kann das Erleben von Machtlosigkeit oder eigener Unzulänglichkeit das Bewusstsein von beruflicher Selbstwirksamkeit unmerklich erodieren. Zweifel an Bedeutung und Sinnhaftigkeit ärztlichen Handelns nehmen zu. Hier setzen Interventionen, z. B. in Form von Kommunikationstrainings, an, die die Selbstwirksamkeit von Onkologen in der Kommunikation mit Patienten fördern, sodass schwierige Gespräche leichter fallen und weniger belastend erlebt werden.

\section{Bessere Kommunikation für Arzt und Patient}

Im Rahmen der KoMPASS-Studie nahmen von 2008 bis 2011 insgesamt 345 onkologisch tätige Ärzte an einem qualifizierten Kommunikationstraining, abgestimmt auf ihre persönlichen Anliegen, teil [mehr unter KoMPASS-O.org]. Vier Monate später beurteilten sie ihre „Selbstwirksamkeit“ in Bezug auf kommunikative Herausforderungen deutlich besser; Zeichen für Burnout (MBI „Depersonalisation“) hatten abgenommen, auch im Vergleich mit einer Kontrollgruppe, während ihre berufliche Zufriedenheit zugenommen hatte. Damit konnte erstmals ein präventiver Effekt von Kommunikationstrainings auf die Entstehung von beruflichen Burnout gezeigt werden, von dem Onkologen und, so ist zu hoffen, Patienten gleichermaßen profitieren.

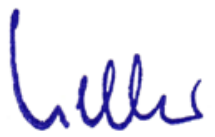

